\title{
Decentralized control with input saturation ${ }^{1}$
}

\author{
Ali Saberi ${ }^{2}$ \\ Anton A. Stoorvogel ${ }^{3}$ \\ Peddapullaiah Sannuti 4
}

\begin{abstract}
In decentralized control it is known that the system can be stabilized only if the so-called fixed modes are all stable. If we have input constraints then (semi-)global stability requires all poles to be in the closed left half plane. This paper establishes that these two requirements are necessary and sufficient for stabilizability of a decentralized system with input saturation.
\end{abstract}

\section{INTRODUCTION}

Nonclassical information and control structure are two essential and distinguishing characteristics of large-scale systems. The research on decentralized control was formally initiated by Wang and Davison in their seminal paper [1] in 1973, and has been the subject of intense study during the 70's and 80's. Most recently there has been a renewed interest in decentralized control because of its fundamental role in the problem of coordinating the motion of multiple autonomous agents which by itself has attracted significant attention. Coordinating the motion of autonomous agents has many engineering applications besides having links to problems in biology, social behavior, statistical physics, and computer graphics. The engineering applications include unmanned aerial vehicles (UAVs), autonomous underwater vehicles (AUVs) and automated highway systems (AHS). A fundamental concept in the study of stabilization using decentralized feedback controllers is that of fixed modes. These are the poles of the system which cannot be shifted just using any type of decentralized linear time-invariant controllers. The idea of fixed modes was introduced by Wang and Davison [1] who also show that decentralized stabilization is possible if and only if the fixed modes are stable. More definitive results are obtained by Corfmat and Morse [2] who present necessary and sufficient conditions under which spectrum assignment is possible in terms of the remnant polynomial of complementary subsystems. Since fixed modes constitute such an important concept in decentralized control, their characterization and determination has been the subject of many papers in the literature.

The majority of existing research in decentralized control makes a critical assumption that the interconnections

\footnotetext{
${ }^{1}$ Support for this work was provided by the Office of Naval Research under grant number N000140310848.

${ }^{2}$ School of Electrical Engineering and Computer Science, Washington State University, Pullman, WA 99164-2752, U.S.A. E-mail: \{saberi,gshi\}@eecs.wsu.edu.

${ }^{3}$ Department of Mathematics, and Computing Science, Eindhoven Univ. of Technology, P.O. Box 513, 5600 MB Eindhoven, The Netherlands. Email: A.A.Stoorvogel@ tue.nl. Department of Electrical Engineering, Mathematics and Computer Science, Delft Univ. of Technology, P.O. Box 5031, 2600 GA Delft, The Netherlands, E-mail: a.a.stoorvogel@ewi.tudelft.nl

${ }^{4}$ Department of Electrical and Computer Engineering, Rutgers University, 94 Brett Road, Piscataway, NJ 08854-8058, U.S.A. E-mail: sannuti@ece.rutgers.edu
}

between the subsystems of a given system are unknown but have known bounds. In this regard, tools borrowed from robust control theory and Lyapunov theory are used for the purpose of either synthesis or analysis of decentralized controllers [3]-[5]. For the case when the interconnections between the subsystems are known, the existing research is very sparse. In fact, in any case, beyond the decentralized stabilization, no results are yet available dealing with the fundamental control issues such as exact or almost disturbance decoupling, control for various performance objectives etc.

From a different perspective, input saturation in any control scheme is a common phenomenon. Every physically conceivable actuator has bounds on its output. Valves can only be operated between fully open and fully closed states, pumps and compressors have a finite throughput capacity and tanks can only hold a certain volume. Ignoring such saturation effects in any control system design can be detrimental to the stability and performance of controlled systems. A classical example for the detrimental effect of neglecting actuator constraints is the Chernobyl unit 4 nuclear power plant disaster in 1986 [6]. During the last decade and the present one, there has been an intense research activity in the area of control of linear plants with saturating actuators. Such intense research activity has been chronicled in special issues of journals and edited books (e.g. for recent literature, see [7] or [8]). Fundamental fuel behind such a research activity has been to accentuate the industrial and thus the practical engineering relevance of modern control theory. In this regard, the primary focus of the research activity has been to take into account $a$ priori the presence of saturation nonlinearities in any control system analysis and design. A number of control issues have been considered so far including internal, external, or internal plus external stabilization and output regulation among others. Although not all aspects of these issues have been completely resolved, it is fair to say that a good understanding of these issues exists at present. However, issues related to performance, robustness etc., are very poorly understood and still remain as challenging and complex problems for future research.

Having been involved deeply in the past with research on linear systems subject to constraints on its input and state variables, we are now ready to open up a new front line of research in decentralized control by bringing into picture the constraints of actuators. The focus of this paper is to determine the necessary and sufficient conditions for decentralized stabilization of linear systems subject to constraints on actuators. Obviously, this is related to the seminal work of Wang and Davison [1] but goes beyond it 
by bringing into picture the input constraints on the top of decentralized constraint.

\section{PROBLEM FORMULATION}

Consider the following system:

$$
\Sigma:\left\{\begin{array}{l}
\dot{x}=A x+\sum_{i=1}^{v} B_{i} \sigma\left(u_{i}\right) \\
y_{i}=C_{i} x \quad(i=1, \ldots, v)
\end{array}\right.
$$

where $\sigma$ denotes the standard saturation element with the property that, for any vector $u$ of arbitrary dimension, $\sigma(u)$ is a vector of the same dimension as $u$ such that for any positive integer $j$ less than or equal to the dimension of $u$ we have

$$
(\sigma u)_{j}= \begin{cases}1 & \text { if } 1<(u)_{j}, \\ (u)_{j} & \text { if }-1 \leq(u)_{j} \leq 1, \\ -1 & \text { if }(u)_{j}<-1,\end{cases}
$$

where $(\sigma u)_{j}$ denotes the $j$ 'th component of $\sigma(u)$ and $(u)_{j}$ denotes the $j$ 'th component of $u$.

We are looking for $v$ nonlinear controllers of the form

$$
\Sigma_{i}:\left\{\begin{array}{l}
\dot{z}_{i}=f_{i}\left(z_{i}, y_{i}\right), \quad z_{i} \in \mathbb{R}^{s_{i}} \\
u_{i}=h_{i}\left(z_{i}, y_{i}\right)
\end{array}\right.
$$

with $f_{i}$ and $h_{i}$ continuously differentiable.

\section{MAIN RESULT}

The important concept of decentralized fixed modes was introduced by Wang and Davison in [1] and is instrumental in the analysis of the problems in this paper.

Definition III.1 Consider a system of the form (1). $\lambda$ is called a decentralized fixed mode of the system if for all matrices $K_{1}, \ldots, K_{v}$ we have that $\lambda$ is an eigenvalue of

$$
A+\sum_{i=1}^{v} B_{i} K_{i} C_{i}
$$

After the introduction of this concept, there has been quite some research on interpretations of this concept. The crucial step in understanding fixed modes was its connection to complementary systems as introduced by Corfmat and Morse in the paper [2]. The paper [9] by Anderson and Clements used the ideas of Corfmat and Morse to yield the following characterization of fixed modes

Lemma III.2 Consider the system (1). We define

$$
B=\left(\begin{array}{lll}
B_{1} & \cdots & B_{v}
\end{array}\right), \quad C=\left(\begin{array}{c}
C_{1} \\
\vdots \\
C_{v}
\end{array}\right) .
$$

Then $\lambda$ is a decentralized fixed mode if and only if at least one of the following three conditions is satisfied:

- $\lambda$ is an uncontrollable eigenvalue of $(A, B)$

- $\lambda$ is an unobservable eigenvalue of $(C, A)$
- There exists a partition of the integers $\{1,2, \ldots, v\}$ into two disjoint sets $\left\{i_{1}, \ldots, i_{m}\right\}$ and $\left\{j_{1}, \ldots, j_{v-m}\right\}$ for which we have

$$
\operatorname{rank}\left(\begin{array}{cccc}
\lambda I-A & B_{i_{1}} & \cdots & B_{i_{m}} \\
C_{k, j_{1}} & 0 & \cdots & 0 \\
\vdots & \vdots & \ddots & \vdots \\
C_{k, j_{v-m}} & 0 & \cdots & 0
\end{array}\right)<n .
$$

Basically decentralized fixed modes are therefore common blocking zeros of a complementary system which are both unobservable and uncontrollable for this complementary system. For a detailed investigation of blocking zeros we refer to the paper [10]. Other attempts to characterize decentralized fixed modes can be found in for instance [11]-[13]. It is important to stress one important factor: fixed modes in decentralized control are derived only for linear systems. It is known that for time-varying linear or nonlinear controllers only a subset of the fixed modes are truly fixed modes in the sense that no decentralized controller can change these modes. For nonlinear, timeinvariant controllers it is not known what can be achieved.

Using this concept of decentralized fixed modes, we will establish in this paper the following characterization of semi-global stabilizability of decentralized linear systems with input saturation:

Theorem III.3 Consider the system $\Sigma$ given by (1). There exists nonnegative integers $s_{1}, \ldots, s_{v}$ such that for all compact sets $\mathcal{W}$ and $s_{i} \subset \mathbb{R}^{s_{i}}(i=1, \ldots v)$ there exist $v$ controllers of the form (2) such that the origin of the resulting closed loop system is locally exponentially stable and the domain of attraction includes $W \times \varsigma_{1} \times \cdots \times s_{v}$ if and only if

- All fixed modes are in the open left half plane,

- All eigenvalues of $A$ are in the closed left half plane.

\section{PRoOf OF Theorem III. 3}

\section{A. Necessity}

We will first show that the conditions of Theorem III.3 are necessary. Assume that we have a family of controllers satisfying the conditions of Theorem III.3. Local exponential stability implies that the linearization of our controllers must achieve local asymptotic stability when applied to the system (1). Clearly locally the saturation elements in (1) do not play a role and hence we have a linear decentralized controller asymptotically stabilizing a linear system. Let these controllers be of the form:

$$
\tilde{\Sigma}_{i}:\left\{\begin{array}{l}
\dot{z}_{i}=K_{i} z_{i}+L_{i} y_{i}, \quad z_{i} \in \mathbb{R}^{s_{i}} \\
u_{i}=M_{i} z_{i}+N_{i} y_{i} .
\end{array}\right.
$$

Then, we define 


$$
\begin{aligned}
K & =\left(\begin{array}{cccc}
K_{1} & 0 & \cdots & 0 \\
0 & K_{2} & \ddots & \vdots \\
\vdots & \ddots & \ddots & 0 \\
0 & \cdots & 0 & K_{\nu}
\end{array}\right), L=\left(\begin{array}{cccc}
L_{1} & 0 & \cdots & 0 \\
0 & L_{2} & \ddots & \vdots \\
\vdots & \ddots & \ddots & 0 \\
0 & \cdots & 0 & L_{v}
\end{array}\right), \\
M & =\left(\begin{array}{cccc}
M_{1} & 0 & \cdots & 0 \\
0 & M_{2} & \ddots & \vdots \\
\vdots & \ddots & \ddots & 0 \\
0 & \cdots & 0 & M_{\nu}
\end{array}\right), N=\left(\begin{array}{cccc}
N_{1} & 0 & \cdots & 0 \\
0 & N_{2} & \ddots & \vdots \\
\vdots & \ddots & \ddots & 0 \\
0 & \cdots & 0 & N_{v}
\end{array}\right) .
\end{aligned}
$$

For any $\lambda$ with $\operatorname{Re} \lambda \geq 0$ there exists a $\delta$ such that $(\lambda+\delta) I-K$ is invertible and the closed loop system when replacing $K$ by $K-\delta I$ is still asymptotically stable. But then the linearization of the closed loop system cannot have a pole in $\lambda$ which implies that we must have

$$
\operatorname{det}\left(\lambda I-A-B\left[M(\lambda I-(K-\delta I))^{-1} L+N\right] C\right) \neq 0 .
$$

But then the block diagonal matrix

$$
S=M(\lambda I-(K-\delta I))^{-1} L+N
$$

has the property that

$$
\operatorname{det}(\lambda I-A-B S C) \neq 0,
$$

and hence $\lambda$ is not a fixed mode of the system. Since this argument is valid for any $\lambda$ in the closed right half plane this implies that all fixed modes must be in the open left half plane.

Next assume that $\lambda$ is an eigenvalue of $A$ in the open right half plane with corresponding left eigenvector $p$, i.e. $p^{\prime} A=\lambda p$. Then we have

$$
\frac{\mathrm{d}}{\mathrm{d} t} p^{\prime} x(t)=\lambda p^{\prime} x(t)+v(t)
$$

and there exists an $M>0$ such that

$$
v(t):=\sum_{i=1}^{v} p^{\prime} B \text { sat } u_{i}(t)
$$

has the property that $\|v(t)\| \leq M$ for all $t>0$. But then

$$
\left|p^{\prime} x(t)\right|>e^{\lambda t}\left(\left|p^{\prime} x(0)\right|-\frac{M}{\operatorname{Re} \lambda}\right)+\frac{M}{\operatorname{Re} \lambda}
$$

which doesnot converge to zero provided the initial condition is such that

$$
\left|p^{\prime} x(0)\right|>\frac{M}{\operatorname{Re} \lambda} .
$$

Note that this is valid for all controllers and therefore clearly we cannot achieve semi-global stability.

\section{B. Sufficiency}

The next step is to prove sufficiency of the conditions in Theorem III.3. For this conference version of the paper we will only establish sufficiency for the case that all the eigenvalues of $A$ are on the imaginary axis and have multiplicity 1 . For this case we will exploit the following lemma which follows directly from classical results of eigenvalues and eigenvectors and the results of perturbations of the matrix on those eigenvalues and eigenvectors (see for instance [14], [15]).

Lemma IV.1 . Let $A \in \mathbb{R}^{n \times n}$ be a matrix with all eigenvalues in the closed left-half plane and with $r$ eigenvalues on the imaginary axis with all of them having multiplicity 1 . Then there exists a matrix $P>0$ such that $A^{\prime} P+P A \leq 0$ and the rank of $A^{\prime} P+P A$ equals $n-r$.

Consider a family of perturbations $\Delta_{\varepsilon}$ parameterized in $\varepsilon$ with $\Delta_{\varepsilon} \rightarrow 0$ as $\varepsilon \downarrow 0$ and such that $A+\Delta_{\varepsilon}$ still has all eigenvalues in the closed left-half plane.

Then for $\varepsilon$ small enough there exists a matrix $P_{\varepsilon}$ such that $\left(A+\Delta_{\varepsilon}\right)^{\prime} P+P\left(A+\Delta_{\varepsilon}\right) \leq 0$ and the rank of $\left(A+\Delta_{\varepsilon}\right)^{\prime} P+P\left(A+\Delta_{\varepsilon}\right)$ equals the number of asymptotically stable eigenvalues of $A+\Delta_{\varepsilon}$. Finally, $P_{\varepsilon} \rightarrow P$ as $\varepsilon \downarrow 0$.

We will present recursively an algorithm which at each step applies a preliminary feedback which stabilizes at least one eigenvalue on the imaginary axis while preserving the stability of the system and such that the magnitude of each preliminary feedback is guaranteed never to exceed $1 / n$. Therefore, after at most $n$ steps the combination of these preliminary feedbacks will asymptotically stabilize the system without ever violating the magnitude constraints of each of the inputs.

We first initialize our algorithm at step 0 . Since the eigenvalues on the imaginary axis all have multiplicity 1 , we know that there exists a matrix $P>0$ such that

$$
A^{\prime} P+P A \leq 0
$$

such that

$$
\operatorname{rank}\left(A^{\prime} P+P A\right)
$$

equals the number of asymptotically stable eigenvalues of A. We define

$$
\mathcal{P}_{0}:=\left\{x \in \mathbb{R}^{n} \mid x^{\prime} P x \leq c\right\}
$$

with $c>0$ such that $\mathfrak{W} \subset \mathcal{P}_{0}$. Note that $\mathcal{P}_{0}$ is an invariant set of the differential equation $\dot{x}=A x$ in the sense that $x(0) \in \mathcal{P}_{0}$ implies that $x(t) \in \mathcal{P}_{0}$ for all $t>0$. Define $A_{0}=$ $A, B_{0, i}=B_{i}$ and $C_{0, i}=C_{i}(i=0, \ldots, v)$. Moreover, $x_{0}=x$.

Consider, the system after $k$ steps. Let $\lambda$ be an eigenvalue on the imaginary axis of $A_{k}$. We know that preliminary feedbacks do not change the fixed modes and therefore, since $\lambda$ was not a fixed mode of the original system, it is 
not a fixed mode of the system obtained after $k$ preliminary feedbacks either. Hence there exists a $\bar{K}_{i}$ such that

$$
A_{k}+\sum_{i=1}^{v} B_{k, i} \bar{K}_{i} C_{k, i}
$$

has no eigenvalue in $\lambda$. Hence

$$
A_{\varepsilon}=A_{k}+\varepsilon \sum_{i=1}^{v} B_{k, i} \bar{K}_{i} C_{k, i}
$$

has no eigenvalue in $\lambda$ for almost all $\varepsilon>0$ (the determinant of $\lambda I-A_{\varepsilon}$ is a polynomial in $\varepsilon$ which is nonzero for $\varepsilon=1$ and hence has a finite number of zeros). Choose $\varepsilon$ small enough such that $K_{i}=\varepsilon \bar{K}_{i}$ has the property that

$$
A_{k}+\sum_{i=1}^{v} B_{k, i} K_{i} C_{k, i}
$$

has no eigenvalue in $\lambda$, and

$$
\left\|K_{i} x\right\|<\frac{1}{2 n}
$$

for all $x$ with $x^{\prime} P_{k} x \leq c+2 n$. Let $j$ be the largest integer such that

$$
\bar{A}=A_{k}+\sum_{i=1}^{v} B_{k, i} \bar{K}_{i} C_{k, i}
$$

has the same number of eigenvalues on the imaginary axis as $A$. Then

$$
\left(\bar{A}, B_{j+1}, C_{j+1}\right)
$$

has a stabilizable and detectable eigenvalue on the imaginary axis. Choose $V$ such that

$$
V V^{\prime}=I \quad \text { and } \quad \operatorname{ker} V=\left\langle\operatorname{ker} C_{k, j+1} \mid \bar{A}\right\rangle .
$$

We choose the following preliminary feedback

$$
\begin{aligned}
\dot{p} & =A_{s} p+\sum_{i=1}^{v} V B_{k, i} u_{i}+K\left(C_{k, j+1} V^{\prime} p-y_{j+1}\right) \\
u_{j+1} & =F_{\delta} p+v_{j+1}
\end{aligned}
$$

with $p \in \mathbb{R}^{s}$ and where $A_{s}$ is such that $A_{s} V=V \bar{A}$ while $K$ is chosen such that $A_{s}+K C_{k, j+1} V^{\prime}$ is asymptotically stable and for all $\delta$ the matrix $A+B F_{\delta} V$ has at least one eigenvalue less on the imaginary axis and still has all eigenvalues in the closed left half plane and $F_{\delta} \rightarrow 0$ as $\delta \downarrow 0$. Rewriting the resulting system in a new basis consisting of $x$ and $p-V x$ results in

$$
\begin{aligned}
\dot{x}_{k+1} & =\left(\begin{array}{cc}
A+B_{j+1} F_{\delta} V & 0 \\
0 & A+K C_{j+1}
\end{array}\right) x_{k+1}+\sum_{i=1}^{v} B_{k+1, i} u_{i} \\
y_{i} & =C_{k+1, i} x_{k+1} \quad(i=1, \ldots, v)
\end{aligned}
$$

for suitably chosen $B_{k+1, i}$ and $C_{k+1, i}$ where

$$
x_{k+1}=\left(\begin{array}{c}
x_{k} \\
p-V x
\end{array}\right) \text {. }
$$

Note that after applying the preliminary feedback, we renamed $v_{j+1}$ as $u_{k+1}$. Strictly speaking not correct but it eases the notation. Choosing any compact set $s_{k} \in \mathbb{R}^{s}$, there exists a matrix $R_{k}>0$ such that

$$
\left(A+K C_{k, j+1}\right)^{\prime} R_{k}+R_{k}\left(A+K C_{k, j+1}\right)<0
$$

and such that for all $x_{k} \in \mathcal{P}_{k}$ and for all $p \in \delta$ we have that

$$
x_{k+1} \in\left\{x \in \mathbb{R}^{n} \mid x^{\prime}\left(\begin{array}{cc}
P_{k} & 0 \\
0 & R_{k}
\end{array}\right) x \leq c+k\right\} .
$$

$A+B_{j+1} F_{\delta} V$ is a perturbation of $A$ which has at least one eigenvalue less on the imaginary axis and still has all eigenvalues in the closed left half plane. But then for $\delta$ small there exists a matrix $\bar{P}_{\delta}$ by Lemma IV.1 such that

$$
\left(A+B_{j+1} F_{\delta} V\right)^{\prime} \bar{P}_{\delta}+\bar{P}_{\delta}\left(A+B_{j+1} F_{\delta} V\right) \leq 0
$$

while

$$
\operatorname{rank}\left[\left(A+B_{j+1} F_{\delta} V\right)^{\prime} \bar{P}_{\delta}+\bar{P}_{\delta}\left(A+B_{j+1} F_{\delta} V\right)\right]=n-r
$$

with $r$ the number of eigenvalues on the imaginary axis of $A+B_{j+1} F_{\delta} V$. Define

$$
P_{k+1}=\left(\begin{array}{cc}
\bar{P}_{\delta} & 0 \\
0 & R_{k}
\end{array}\right) .
$$

Choose a $\delta$ small enough such that the resulting $P_{k+1}$ guarantees that

$$
x_{k+1}^{\prime} P_{k+1} x_{k+1} \leq c+2 n-k-1
$$

implies

$$
x_{k}^{\prime} P_{k} x_{k} \leq c+2 n-k
$$

Moreover

$$
\left\|F_{\delta} x\right\| \leq \frac{1}{2 n}
$$

for all $x$ satifying $x_{k+1}^{\prime} P_{k+1} x_{k+1} \leq c+2 n$. Finally

$$
\mathcal{P}_{k+1} \in\left\{x \in \mathbb{R}^{n} \mid x^{\prime} P_{k+1} x \leq c+k+1\right\}
$$

is such that $x_{k} \in \mathscr{P}_{k}$ and $p \in \delta_{k}$ implies that $x_{k+1} \in \mathcal{P}_{k+1}$.

Next set $k=k+1$ and stop if $A_{k+1}$ is asymptotically stable. Otherwise repeat the above to remove a further imaginary axis eigenvalue.

But then after the final step we have an asymptically stable system with an invariant set $\mathcal{P}_{k+1}$ such that for all initial conditions of the original system in $\mathcal{P}_{0}$ and all initial conditions of the preliminary feedbacks in the sets $\bar{\gamma}_{i}$ we have that the state is in $\mathcal{P}_{k+1}$ and each preliminary feedback is such that the magnitude of the input is no larger than $1 / n$. Hence all these preliminary feedbacks together will never cause a constraint violation. This concludes the proof.

\section{CONClusion}

Decentralized control is an important problem in many applications. The same is true for input saturation. This is one of the first papers which looks at the intricacies of the link between saturation and decentralized control. Clearly, there are still many open problems to be resolved. Stabilization is a first step but the aim is obviously to come to a design methodology for decentralized controllers subject to saturation. 


\section{REFERENCES}

[1] S. Wang and E. Davison, "On the stabilization of decentralized control systems," IEEE Trans. Aut. Contr., vol. 18, no. 5, pp. 473478, 1973.

[2] J. Corfmat and A. Morse, "Decentralized control of linear multivariable systems," Automatica, vol. 12, no. 5, pp. 479-495, 1976.

[3] A. Michel and R. Miller, Qualitative analysis of large scale dynamical systems. Academic Press, 1977.

[4] D. Siljak, Decentralized control of complex systems. London: Academic Press, 1991.

[5] _ Large-scale dynamic systems : stability and structure. Amsterdam: North Holland, 1978.

[6] G. Stein, "Respect the unstable," in IEEE Conference of Decision and Control, Tampa, FL, 1989, bode prize lecture.

[7] V. Kapila and G. Grigoriadis, Eds., Actuator saturation control. Marcel Dekker, 2002.

[8] A. Saberi and A. Stoorvogel, Eds., "Special issue on control problems with constraints," Int. J. Robust \& Nonlinear Control, vol. 9, no. 10, pp. 583-734, 1999.

[9] B. Anderson and D. Clements, "Algebraic characterization of fixed modes in decentralized control," Automatica, vol. 17, no. 5, pp. 703$712,1981$.

[10] B. Chen, A. Saberi, and P. Sannuti, "On blocking zeros and strong stabilizability of linear multivariable systems," Automatica, vol. 28, no. 5, pp. 1051-1055, 1992 .

[11] H. Seraji, "On fixed modes in decentralized control systems," Int. J. Contr., vol. 35, no. 5, pp. 775-784, 1982.

[12] Z. Gong and M. Aldeen, "On the characterization of fixed modes in decentralized control," IEEE Trans. Aut. Contr, vol. 37, no. 7, pp. 1046-1050, 1992.

[13] N. Gündeş and C. Desoer, Algebraic theory of linear feedback systems with full and decentralized compensators, ser. Lecture Notes in Control and Information Sciences. Springer Verlag, 1990, vol. 142.

[14] T. Kato, Perturbation theory for linear operators, 2nd ed. Berlin: Springer Verlag, 1976.

[15] G. Golub and C. van Loan, Matrix Computations, 2nd ed. John Hopkins Univ. Press, 1989. 